\title{
Proteomic Applications of Polymeric Nanoparticles with Engineered Affinity towards Select Target
}

\begin{abstract}
Complexity in biological samples pose a greater challenge for proteomic analysis. In order to overcome this issue, separation or fractionation is often a prerequisite to qualitative or quantitative proteomic approaches. Affinity chromatography is a method for separating biochemical mixture based on a highly specific interaction similar to the one between receptor and ligand. This mini-review discuss the use of polymeric nanoparticles as an alternative matrix support in affinity purification. The nanoparticle-based purification have the potential to selectively target the protein of interest from a complex pool. This strategy could be applied as sample purification step prior to mass spectrometry-based proteomic analyses.
\end{abstract}

Keywords: Polymeric nanoparticles, Proteomics, Affinity purification, Mass spectrometry
Volume 5 Issue 4 - 2017

\author{
Hariprasad Thangavel,' Dhanya Dhanyalayam² \\ 'Department of Biology and Biochemistry, University of \\ Houston, USA \\ ${ }^{2}$ Department of Pharmacy, Health and Nutritional Sciences, \\ University of Calabria, Italy \\ Correspondence: Hariprasad Thangavel, Center for Nuclear \\ Receptors and Cell Signaling, Department of Biology and \\ Biochemistry, University of Houston, Houston, TX 77204, USA, \\ Tel 832-230-6465 Email hariprasadsIm@gmail.com
}

Received: April 2I, 2017 | Published: May II, 2017
Abbreviations: MS, Mass Spectrometry; NPs, Nanoparticles; PNIPAm, poly-N-isopropylacrylamide; GPER, G-protein coupled Estrogen Receptor; HPLC, High Performance Liquid Chromatography

\section{Introduction to affinity purification}

Proteins and other macromolecules of interest can be purified from crude extracts or other complex mixtures by a variety of methods. Selective precipitation is perhaps the simplest method for separating one type of macromolecule from another. Most purification methods, however, involve some form of chromatography whereby molecules in solution (mobile phase) are separated based on differences in chemical or physical interaction with a stationary material (solid phase). Gel filtration (also called size-exclusion chromatography or SEC) uses a porous resin material to separate molecules based on size (i.e., physical exclusion). In ion exchange chromatography, molecules are separated according to the strength of their overall ionic interaction with a solid phase material (i.e., nonspecific interactions).

By contrast, affinity chromatography (also called affinity purification) makes use of specific binding interactions between molecules. It is a variant of chromatography based on the ability of biomolecules (analytes) to bind certain ligands specifically and reversibly. These unique features of the analyte and the ligand interaction are then utilized for the separation of the analyte of interest from a complex mixture. From the first protein-protein interaction studies done in the late $1990^{\prime} \mathrm{s},{ }^{1,2}$ affinity separations have experienced a true renaissance in proteomics. A complete parade of affinity matrices and affinity-based experimental approaches has been developed that has found numerous applications ranging from subtraction of highly abundant proteins to study of drug target profiles to large scale mapping of posttranslational modifications. In the classical setup, a relevant ligand is attached to a solid, inert resin creating an affinity stationary phase (affinity matrix). When a sample containing desired analyte is passed over such affinity matrix, the analyte having specific binding affinity to the ligand become bound and retained by the matrix while the other molecules stay apart. After the other unwanted molecules are washed away, the bound analyte is stripped from the affinity matrix, resulting in its purification from the original sample (Figure 1). This principle was discovered by Cuatrecasas and Wilchek, ${ }^{3,4}$ who applied it to the purification of Staphylococcal nuclease and avidine. Since their discovery, numerous specialized affinity purification techniques appeared, but notably even today more than $90 \%$ of them apply the same general principles as reported in $1968 .{ }^{5}$ Each specific affinity system requires its own set of conditions and presents its own peculiar challenges for a given research purpose. ${ }^{6}$

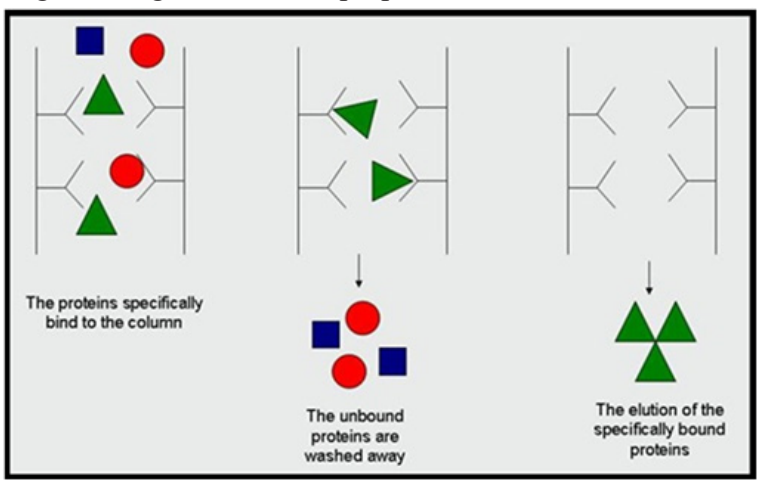

Figure I Protein purification using affinity chromatography. ${ }^{7}$

Basically, there are two main modes of affinity chromatography - a 'subtraction' mode and an 'enrichment' mode. If the aim of the purification is to specifically remove protein species that would hamper characterization of the sample, then affinity chromatography is employed in subtraction mode. Traditionally, the main purpose of affinity subtraction is the elimination of highly abundant proteins like albumin, immunoglobulin, etc., to achieve broader coverage of proteomes that suffer from wide dynamic range such as human body fluids (blood plasma, cerebrospinal fluid, urine, saliva). Whereas, enrichment mode is applied for the isolation of selected protein species. At the protein level, the enrichment affinity chromatography permits the purification of a particular protein of interest or a group of low abundant proteins and/or proteins that share a specific structural feature. At peptide level, the enrichment affinity chromatography has attained an essential position in the purification of post-translationally modified species.

Moreover, the enrichment affinity chromatography of proteins can be used as a tool to obtain an information on specific protein affinities. In short, the basic principle of affinity chromatography allows to utilize the method for the isolation of protein partners of selected 
molecules. ${ }^{7,8}$ During the affinity purification, the molecule of interest represents a 'bait' that is bound by its cellular protein counterparts, its 'preys'. These (protein) preys are then easily purified and consequently identified by MS, ${ }^{9,10}$ generating thus a map of the baitprotein interaction network. In this setup, affinity chromatography has facilitated the discernment of many different molecular relationships from protein-protein interactions to drug selectivity profiles. ${ }^{11-13}$

\section{Engineered polymeric nanoparticles for affinity purification}

The support or matrix in affinity purification is any material to which a bio-specific ligand is covalently attached. Typically, the material to be used as an affinity matrix is insoluble in the system in which the target molecule is found. Usually, but not always, the insoluble matrix is a solid. Hundreds of substances have been described and utilized as affinity matrices, including agarose, cellulose, dextran, polyacrylamide, latex and controlled pore glass. ${ }^{14-16}$ Useful affinity supports are those with a high surface-area to volume ratio, chemical groups that are easily modified for covalent attachment of ligands, minimal nonspecific binding properties, good flow characteristics and mechanical and chemical stability.

Over the last decade, the use of magnetic nanoparticles ${ }^{17-20}$ and polymer nanoparticles ${ }^{21-24}$ for protein affinity purification have been widely reported. Engineered synthetic nanoparticles (NPs) with an intrinsic affinity and selectivity for target biomacromolecules are significant interest for use in diagnostics ${ }^{25}$ therapeutics ${ }^{26-28}$ and protein purification, ${ }^{29-30}$ and as a tool to investigate biochemical processes. ${ }^{31-32}$ Recent studies show that synthetic NPs (Figure 2) incorporating functional groups complementary to a surface domain of a target biomacromolecule can result in a high intrinsic affinity for target peptides, ${ }^{28}$ proteins, ${ }^{26,33}$ and polysaccharides. ${ }^{34,35}$ These materials are attractive as an inexpensive and robust alternative to affinity reagents of biological origin, including antibodies. ${ }^{36}$

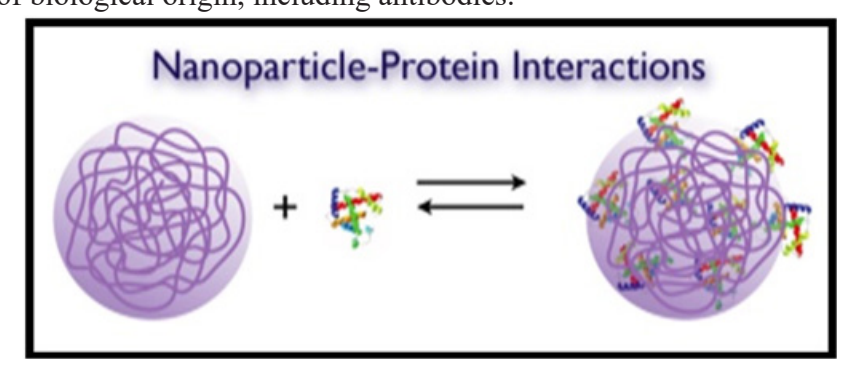

Figure 2 Synthetic polymer nanoparticles for protein purification.

\section{PNIPAm-based nanoparticles for GPER purification}

G-protein coupled Estrogen Receptor may play a significant role in tamoxifen resistance in breast cancer cells. Although it is believed to be a key player in several other cancers, the background information at the molecular level is still limited. In order to study the complete proteome, post translational modifications, mutations and to better understand their molecular interactions with various other receptors and ligands, it is necessary to isolate pure GPER from crude cell lysate. The lack of promising separation and purification tools peaked our interest towards designing NPs that can capture this target biomolecule. In recent years, NPs with an intrinsic affinity have shown to be successful in binding biomacromolecules like melittin, ${ }^{37,38}$ immunoglobulin $\mathrm{G},{ }^{39}$ histone, ${ }^{40}$ fibrinogen ${ }^{40}$ and lysozyme ${ }^{29}$ by controlling and optimizing the functional monomers composition.

In our study, we adopted a similar approach in an effort to capture GPER with high affinity and selectivity among a mixture of proteins that are expressed in various breast cancer cell lines. Considering the fact that GPER is a membrane protein with many hydrophobic amino acid residues on the surface, a candidate NP was chosen from a library of nanoparticles that were prepared by combining different populations of functional groups on a poly-N-isopropylacrylamide (PNIPAm)-based polymer backbone. The synthesis of the candidate NPs was illustrated below (Figure 3). We started our experiment with GPER peptide selection and synthesis. Then, we evaluated the interaction between truncated-GPER (short peptide epitopes) and NPs by high performance liquid chromatography (HPLC). Finally, we moved on to evaluate the interaction between GPER (whole protein) and NPs by immunoblot analysis. After evaluating the results, the nanoparticle-based purification approach turned out to be the method of choice to enrich GPER prior to mass spectrometry analysis.

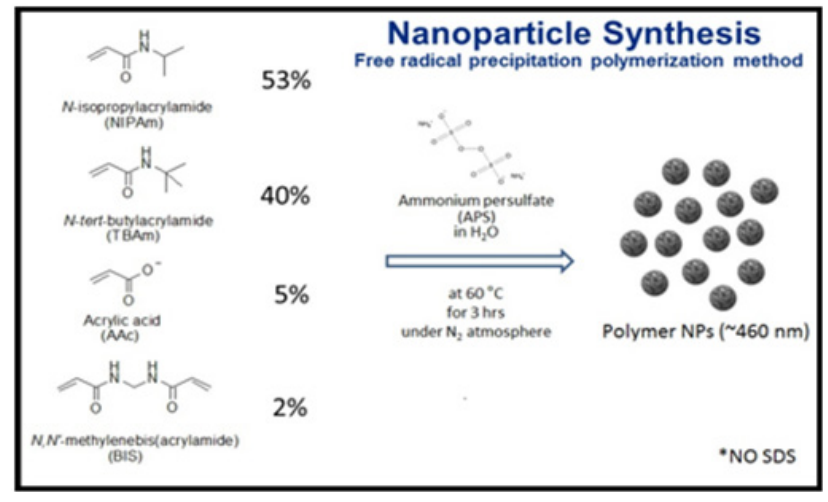

Figure 3 Synthesis of PNIPAm-based NPs with affinity towards GPER.

\section{Conclusion}

Recently, nanoparticles with an intrinsic affinity have shown to be successful in binding biomacromolecules like melittin, immunoglobulin $\mathrm{G}$, histone, fibrinogen and lysozyme by controlling and optimizing the functional monomers composition. We tested a similar approach in an effort to capture GPER with high affinity and selectivity among a mixture of proteins that are expressed in various cancer cell lines. We anticipate to use this strategy as a sample purification step before mass spectrometry-based proteomic analysis. Similar approach could be applied to study a number complex targets that are difficult to purify or lack separation tools with current proteomic technologies.

\section{Acknowledgments}

I would like to acknowledge Prof. Kenneth J. Shea, University of California, Irvine for providing the opportunity to learn nanoparticle synthesis in his lab. The research work was supported by the European commission under grant agreement number 264772 (ITNCHEBANA).

\section{Conflicts of interest}

None.

\section{References}

1. Neubauer G, Gottschalk A, Fabrizio P, et al. Identification of the proteins of the yeast U1 small nuclear ribonucleoprotein complex by mass spectrometry. Proc Natl Acad Sci USA. 1997;94(2):385-390.

2. Gitte Neubauer, Angus King, Juri Rappsilber, et al. Mass spectrometry and EST-database searching allows characterization of the multiprotein spliceosome complex. Nat Genet. 1998;20(1):46-50. 
3. Cuatrecasas P, Wilchek M, Anfinsen CB. Selective enzyme purification by affinity chromatography. Proc Natl Acad Sci USA. 1968;61(2):636-643.

4. Cuatrecasas P, Wilchek M. Single-step purification of avidine from egg white by affinity chromatography on biocytin-Sepharose columns. Biochem Biophys Res Commun. 1968;33(2):235-239.

5. M Wilchek. My life with affinity. Protein Sci. 2004;13(11):3066-3070.

6. Cuatrecasas P, Anfinsen CB. Affinity Chromatography. Annual Review of Biochemistry. 1971;40:259-278.

7. Tőzsér J, Emri T, Csősz É. Protein purification (chromatographic techniques) and analysis (SDS-PAGE, 2DE, mass spectrometry). Protein Biotechnology, Hungary. 2011.

8. Muronetz VI, Sholukh M, Korpela T. Use of protein-protein interactions in affinity chromatography. $J$ Biochem Biophys Methods. 2001;49(1-3):29-47.

9. Bauer A, Kuster B. Affinity purification-mass spectrometry: Powerful tools for the characterization of protein complexes. Eur J Biochem. 2003;270(4):570-578.

10. Dunham WH, Mullin M, Gingras AC. Affinity-purification coupled to mass spectrometry: Basic principles and strategies. Proteomics. 2012;12(10):1576-1590.

11. Belanger KD. Using affinity chromatography to investigate novel protein-protein interactions in an undergraduate cell and molecular biology lab course. CBE Life Sci Educ. 2009;8(3):214-225.

12. Hutchens TW, Yip TT, Porath J. Protein interaction with immobilized ligands: Quantitative analyses of equilibrium partition data and comparison with analytical chromatographic approaches using immobilized metal affinity adsorbents. Analytical Biochemistry. 1988;170(1):168-182.

13. Hoffmann A, RG Roeder. Purification of his-tagged proteins in nondenaturing conditions suggests a convenient method for protein interaction studies. Nucleic Acids Res. 1991;19(22):6337-6338.

14. Cuatrecasas P. Protein Purification by Affinity Chromatography: derivatizations of agarose and polyacrylamide beads The Journal of Biological Chemistry. 1970;245:3059-3065.

15. Porath J. Immobilized metal ion affinity chromatography. Protein Expression and Purification. 1992;3(4):263-281.

16. Porath J, B Olin. Immobilized metal affinity adsorption and immobilized metal affinity chromatography of biomaterials. Serum protein affinities for gel-immobilized iron and nickel ions. Biochemistry. 1983;22(7):1621-1630.

17. Safarik I, Safarikova M. Magnetic techniques for the isolation and purification of proteins and peptides," BioMagnetic Research and Technology. 2004;2(7):1-17.

18. Franzreb F, Siemann-Herzberg M, Hobley TJ, et al. Protein purification using magnetic adsorbent particles. Applied Microbiology and Biotechnology. 2006;70(5):505-516.

19. Peter JF, Otto AM. Magnetic particles as powerful purification tool for high sensitive mass spectrometric screening procedures. Proteomics. 2010;10(4):628-633.

20. Fischer I, Hsu CC, Gärtner M, et al. Continuous protein purification using functionalized magnetic nanoparticles in aqueous micellar twophase systems. J Chromatogr A. 2013;1305:7-16.

21. Kim JH, Yoon JY. Protein adsorption on polymer particles. Surface and Colloid Science, 2002;pp.4373-4381.

22. Hoshino Y, Haberaecker WW, Kodama T, et al. Affinity Purification of Multifunctional Polymer Nanoparticles. $J$ Am Chem Soc. 2010;132(39):13648-13650.
23. Boyer C, X Huang, Whittaker MR, et al. An overview of proteinpolymer particles. Soft Matter. 2011;7:1599-1614.

24. Hoshino Y, Lee H, Y Miura. Interaction between synthetic particles and biomacromolecules: fundamental study of nonspecific interaction and design of nanoparticles that recognize target molecules. Polymer Journal. 2014;46:537-545.

25. Saha K, Agasti SS, Kim C, et al. Gold nanoparticles in chemical and biological sensing. Chem Rev. 2012;112(5):2739-2779.

26. Khandare J, Calderón M, Dagia NM, et al. Multifunctional dendritic polymers in nanomedicine: opportunities and challenges. Chem Soc Rev. 2012;41(7):2824-2848.

27. Smith MH, Lyon LA. Multifunctional nanogels for siRNA delivery. Acc Chem Res. 2012;45(7):985-993.

28. Hoshino Y, Koide H, Furuya K, et.al. The rational design of a synthetic polymer nanoparticle that neutralizes a toxic peptide in vivo. Proc Nat Acad Sci U S A. 2012;109(1):33-38.

29. Yoshimatsu K1, Lesel BK, Yonamine Y, et al. Temperature-Responsive "Catch and Release" of Proteins by using Multifunctional PolymerBased Nanoparticles. Angew Chem Int Ed Engl. 2012;51(10):2405-2408.

30. Yonamine Y, Yoshimatsu K, Lee SH, et al. Polymer nanoparticleprotein interface. Evaluation of the contribution of positively charged functional groups to protein affinity. ACS Appl Mater Interfaces. 2013;5(2):374-379.

31. Monopoli MP, C Aberg, A Salvati, et al. Biomolecular coronas provide the biological identity of nanosized materials. Nat Nanotechnol. 2012;7(12):779-786.

32. Kim ST, Saha K, Kim C, et al. The role of surface functionality in determining nanoparticle cytotoxicity. Acc Chem Res. 2013;46(3):681-691.

33. Fischer NO, McIntosh CM, Simard JM, et al. Inhibition of chymotrypsin through surface binding using nanoparticle-based receptors. Proc Natl Acad Sci USA. 2002;99(8):5018-5023.

34. Zeng Z, Patel J, Lee SH, et al. Synthetic Polymer NanoparticlePolysaccharide Interactions: A Systematic Study. $\mathrm{J}$ Am Chem Soc vol. 2012;134(5):2681-2690.

35. Sun W, H Bandmann, T Schrader. A Fluorescent Polymeric Heparin Sensor. Chemistry A European Journal. 2007;13(27):7701-7707.

36. Yoshimatsu K, Yamazaki T, Hoshino Y, et.al. Epitope Discovery for a Synthetic Polymer Nanoparticle: A New Strategy for Developing a Peptide Tag. s. 2014;136(4):1194-1197.

37. Hoshino Y, Urakami T, Kodama T, et.al. Design of synthetic polymer nanoparticles that capture and neutralize a toxic peptide. Small. 2009;5(13):1562-1568.

38. Hoshino Y, Koide H, Urakami T, et.al. Recognition, neutralization and clearance of target peptides in the blood stream of living mice by molecular imprinted polymer nanoparticles: a plastic antibody. $\mathrm{J} \mathrm{Am}$ Chem Soc. 2010;132(19):6644-6645.

39. Shih Hui Lee, Yu Hoshino, Arlo Randall, et al. Engineered synthetic polymer nanoparticles as IgG affinity ligands. Journal of the American Chemical Society. 2012;134(38):15765-15772.

40. Yonamine Y, Hoshino Y, Shea KJ. ELISA-mimic screen for synthetic polymer nanoparticles with high affinity to target proteins. Biomacromolecules. 2012;13(9):2952-2957. 